\title{
Bioanalysis of biotherapeutic proteins and peptides: immunological or MS approach?
}

"When peptide bioanalysis is required, LC-MSIMS could be the analytical method of choice unless the required sensitivity is unattainable ... it remains to be seen whether MS can supplant immunoassays for large-molecule analysis in much the same way that ELISAs supplanted RIAs in the 1980s, but the tools are available."

Keywords: ELISA $=\mathrm{LC}-\mathrm{MS} / \mathrm{MS}$ = peptide bioanalysis " protein bioanalysis

Historically, the bioanalysis of proteins and peptides has been performed using immunological-based techniques. However, continued improvements in MS-based methodologies over the past 10 years have generated assays with sufficient robustness and sensitivity to be applied to the quantitation of biomolecules $[1,2]$. This was evident at the European Bioanalysis Forum (EBF) 'Large Meets Small' meeting in June 2011, where bioanalytical laboratories discussed the current state of the art in protein and peptide bioanalysis by LC-MS/MS [101]. The general outcome of the EBF meeting indicated that LC-MS/MS is a credible technique for quantifying biotherapeutics in biological matrices. This editorial aims to highlight the pros and cons of the two techniques, and raise issues that need to be considered when selecting the most appropriate bioanalytical technique for a given biotherapeutic.

\section{Protein bioanalysis}

The development of increasingly sensitive MS systems, together with the introduction of UHPLC column chemistries, has given us the opportunity to use MS-based methods for the detection of macromolecules. However, the quantitative bioanalysis of biotherapeutic proteins has traditionally been performed using immunological-based techniques, using antibodies raised against the target protein or peptide. The most commonly used methods are ELISA-based, which supplanted radioimmunoassays (RIAs) as the method of choice in the early 1980s. RIAs still have their place where sensitivity is paramount, but immunoassay techniques such as electrochemiluminescence and fluorescence are emerging that offer the ability to multiplex while reducing the sample volume required for analysis. Although the generation of suitable antibodies to a biotherapeutic can take some time, the benefits of using immunological detection methodologies are significant - including highsample throughput, sensitivity and confirmation of a protein's structural stability. Despite these important characteristics, immunologicalbased detection techniques are subject to some equally significant drawbacks, such as obtaining sufficient analyte selectivity, antidrug-antibody interferences and matrix effects. The application of MS-based methodologies, in combination with proteolytic digestion, can address some of these issues affecting immunologicalbased methodologies, and add significant improvements such as multiplexing and fast method development. The relative merits and drawbacks of immunoassay and MS approaches are discussed below.

\section{- Sensitivity}

One of the major differences between the MS and immunological techniques is the sensitivity that can be achieved. Immunological-based methods can detect proteins in complex matrices, such as plasma, down to the low $\mathrm{pg} / \mathrm{ml}$ concentration. MS methodologies can achieve these concentrations, but require significant sample work-up and highly specific extraction methodologies [102]. Furthermore, low $\mathrm{pg} / \mathrm{ml}$ level LC-MS/MS quantitation requires nanoflow systems, which are not in routine use in bioanalytical laboratories. Typically, the required LLOQs for clinical biotherapeutic protein pharmacokinetic (PK) studies are beyond the achievable sensitivity of a high flow rate LC-MS/MS-based methodology. However, in the case of drug discovery and toxicology studies, the relatively high dosing

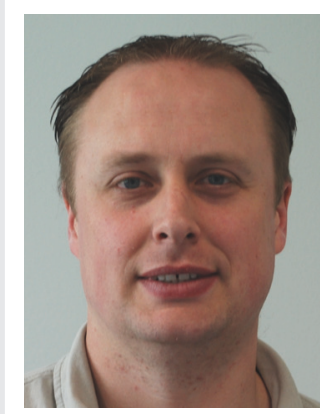

Richard G Kay

Quotient Bioresearch Ltd, Newmarket Road, Fordham, CB7 5WW, UK

Tel.: +44 1638720500

E-mail: richard.kay@

quotientbioresearch.com



Andrew Roberts

Quotient Bioresearch Ltd, Newmarket Road, Fordham, UK 
and concomitant high matrix concentrations of drug makes LC-MS/MS a good candidate for discovery bioanalysis.

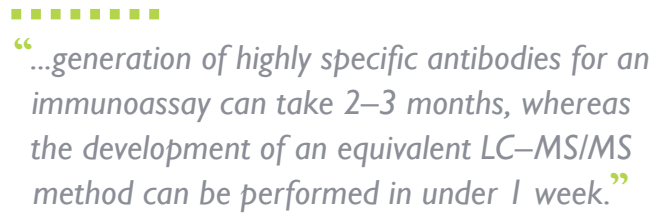

\section{- Analyte selectivity}

Both immunological and LC-MS/MS-based methodologies can be used to differentiate between closely related analytes, although the method development time will differ significantly. The generation of highly specific antibodies for an immunoassay can take 2-3 months, whereas the development of an equivalent LC-MS/MS method can be performed in under 1 week. Furthermore, differences between closely related analytes may be within regions of the protein structure not accessible to antibodies, which makes it virtually impossible to develop an immunoassay-based method to discriminate between the two. Therefore, LC-MS/MS can provide significant advantages where selectivity rather than sensitivity is the overriding consideration.

\section{- Protein structural stability}

The antibody-antigen interaction utilized in immunological-based methodologies requires the antigen to be in its folded state for the antibody to recognise its target epitope. Therefore, most immunological techniques only detect folded protein, conferring analyte stability through detection. The use of a proteolytic digestionbased methodology will involve almost complete enzymatic cleavage of the protein, making structural stability impossible to confirm. Partial-protein stability can be demonstrated through selective preanalytical extraction methodologies (e.g., immunocapture), or monitoring a peptide spanning a specific region where protease-mediated cleavage occurs. Most proteolytic digestion-based LC-MS/MS methodologies will only generate total protein concentrations.

\section{Antidrug-antibody interferences}

A major issue with the development of proteinbased drugs is the propensity to generate antidrug antibodies (ADAs). Apart from any safety considerations of the clinical use of the drug, an ADA response can impact and interfere with immunological-based detection mechanisms. This interference is due to the detection or capture antibody being occluded from their epitopes by antibodies already bound to the drug and can confound any interpretation of exposure to drug. The development of LC-MS/MS-based methodologies can involve treatment of the sample (e.g., by reduction and alkylation) to break antibody interactions thereby releasing the antibodies from the biotherapeutic. This should allow the measurement of total drug in the sample, however, if the LC-MS/MS methodology involves an immunocapture step, then this pre-analytical step might be affected by ADA interactions that also affect immunoassays.

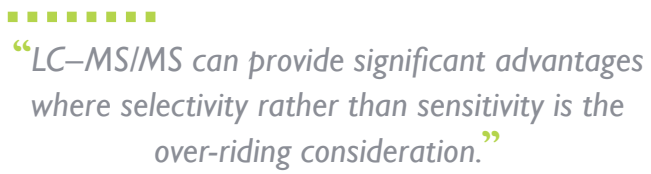

\section{Multiplexing}

LC-MS/MS- and SRM-based methodologies are inherently multiplexed, and when used with software capable of acquiring time-resolved data can detect significant numbers of compounds within a single analysis [103]. In contrast, few immunological-based methodologies include multiplexed detection (e.g., MesoScale Discovery and Luminex), and usually each analyte is measured in an independent assay, thereby significantly increasing analysis costs. However, in the majority of preclinical and clinical PK studies, only a single biotherapeutic will need quantitative measurement, therefore making this LC-MS/MS advantage less applicable.

\section{Peptide bioanalysis}

Biotherapeutic peptides are significantly smaller (and easier to handle) than their larger protein counterparts, and in most cases the peptides can be detected using an LC-MS/MS system without digestion. However, peptides are significantly less stable than proteins in in vivo and in vitro environments, and can undergo rapid metabolism and inactivation by endogenous proteases. For example, peptides such as GLP-I and its analogues, neuropeptide $\mathrm{Y}$ and glucagon [3-5], are rapidly cleaved to inactive metabolites and immunological-based approaches will, in some cases, not be able to differentiate between the parent molecule and one that has been metabolized. The analysis of peptides using an LC-MS/MS-based methodology enables the 
simultaneous selective quantification of both the whole and modified compound. Furthermore an LC-MS/MS-based methodology can discriminate between a drug-peptide analogue and the endogenous peptide counterpart, whereas an immunoassay method may struggle unless the antibody recognises the region of the modifications. However, despite this benefit, the adoption of MS-based methodologies for peptide analysis has been relatively slow, in part because of the difficulty in achieving the same level of sensitivity of immunological-based methods. The main cause of low sensitivity is that the peptide ion current is divided amongst the multiple charge states that are common in electrospray ionization. However, the combination of an efficient extraction method and the use of the most up-todate instrumentation can enable peptides to be detected in the low $\mathrm{pg} / \mathrm{ml}$ concentration range [6], rivaling the sensitivity of immunological-based approaches. Therefore if an LC-MS/MS-based approach has the required sensitivity, it would be the most appropriate quantitation methodology.

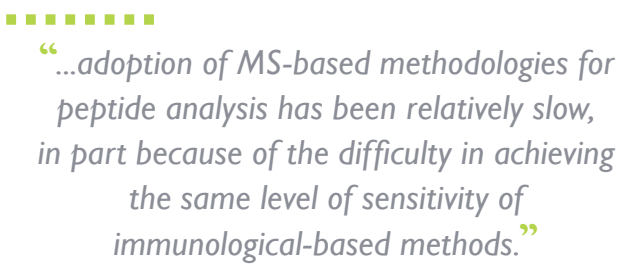

- MS versus immunoassay

Where the two approaches are compared, generally a good correlation between the concentrations is observed [2,7]. In one study in our laboratory, a comparison of the techniques demonstrated good correlation, but poor agreement. Further investigation identified that the ELISA values were being affected by structural stability issues of the protein standard, which only affected the ELISA. In a peptide RIA and LC-MS/MS comparison, the analyte concentration measured by LC-MS/MS was consistently only $5 \%$ of that observed by the RIA. In this case, severe peptide degradation was occurring in plasma after sample collection, which the RIA was not capturing.

\section{Conclusion}

Improvements in LC-MS/MS-based methodologies have increased the available techniques that can be used by bioanalytical laboratories to quantify biotherapeutics. For protein analyses, the application of LC-MS/MS to protein quantitation would be most appropriate in a discovery setting, where rapid method development obviated by the need to develop antibody reagents can be applied to quantify compounds to provide fast turnaround information. Another appropriate use of LC-MS/MS for protein quantitation is where there are significant interferences with an existing immunological-based assay, or insufficient selectivity to differentiate the biotherapeutic protein from endogenous compounds.

When peptide bioanalysis is required, LC-MS/MS could be the analytical method of choice unless the required sensitivity is unattainable. If an immunological-based methodology is needed, then analyte stability should be assessed in both an in vivo and in vitro environment to demonstrate that the assay does not detect metabolized peptide. It remains to be seen whether MS can supplant immunoassays for large-molecule analysis in much the same way that ELISAs supplanted RIAs in the 1980s, but the tools are available.

\section{Financial \& competing interests disclosure}

The authors are employees of Quotient Bioresearch. The authors have no other relevant affiliations or financial involvement with any organization or entity with a financial interest in or financial conflict with the subject matter or materials discussed in the manuscript apart from those disclosed.

No writing assistance was utilized in the production of this manuscript.

\section{References}

1 Kay RG, Gregory B, Grace PB, Pleasance S. The application of ultra-performance liquid chromatography/tandem mass spectrometry to the detection and quantitation of apolipoproteins in human serum. Rapid Commun. Mass Spectrom. 21(16), 2585-2593 (2007).
2 Heudi O, Barteau S, Zimmer D et al. Towards absolute quantification of therapeutic monoclonal antibody in serum by LC-MS/MS using isotope-labeled antibody standard and protein cleavage isotope dilution mass spectrometry. Anal. Chem. 80 (11), 4200-4207 (2008)

(1)

of glucagon-like peptide-1-(7-36) amide after in vivo administration to dogs, and it acts as an antagonist on the pancreatic receptor. Eur. J. Pharmacol. 318, 429-435 (1996).

4 Abid K, Rochat B, Lassahn PG et al. Kinetic study of neuropeptide Y (NPY) proteolysis in blood and identification of NPY3-35. J. Biol. Chem. 284(37), 24715-24724 (2009). 


\section{EDITORIAL | Kay \& Roberts}

5 Hinke SA, Pospisilik JA, Demuth HU et al. Dipeptidyl peptidase IV (DPIV/CD26) degradation of glucagon. J. Biol. Chem. 275(6), 3827-3834 (2000).

6 Lövgren U, Johansson S, Jensen LS et al. Quantitative determination of peptide drug in human plasma samples at low $\mathrm{pg} / \mathrm{ml}$ levels using coupled column liquid chromatographytandem mass spectrometry. J. Pharmaceut. Biomed. Anal. 53, 537-545(2010).

7 Barton C, Kay RG, Gentzer Wet al. Development of high-throughput chemical extraction techniques and quantitative HPLC-MS/MS (SRM) assays for clinically relevant plasma proteins. J. Proteome Res. 9, 333-340 (2010).

\section{- Websites}

101 European Bioanalytical Forum 2nd Focus meeting. Large meets Small. 21-22 June 2011. http://bru2011.europeanbioanalysisforum.eu/ program

102 Shultz G. Advion EBF presentation. Validation of an immunoprecipitation, digestion and immunoaffinity LC-MS/MS assay for human NGF biomarker and implementation in support of clinical trials. http://bru2011.europeanbioanalysisforum.eu
103 Kay RG, Howard JW, Pleasance S. Time-resolved SRM analysis and highly multiplexed LC-MS/MS for quantifying tryptically digested proteins. current trends in mass spectrometry. Chromatography Online (2011).

www.chromatographyonline.com/lcgc/TimeResolved-SRM-Analysis-and-HighlyMultiplexed-/ArticleStandard/Article/ detail/716438 